\title{
Pre-Procedural C-Reactive Protein Level Predicts In-Hospital Outcome in Percutaneous Coronary Intervention
}

\author{
MM Haque, M Ullah, MS Alam, SU Ulabbi, R Uddin \\ National Institute of Cardiovascular Diseases, Dhaka.
}

Keywords:

C-Reactive Protein, In hosital outcome, PCI.

\begin{abstract}
:
Background:To accurately stratify patients undergoing PCI, according to their risk of future adverse events, a quest for risk predictors is ongoing worldwide. But only a few powerful and independent predictors of early and late major adverse cardiovascular events have been found. These include traditional risk factors, such as acute coronary instability, diabetes mellitus, reference vessel diameter, and lesion and/or stent length. This study was undertaken to find out the relationship between pre-procedural CRP level with in-hospital (both procedural and before discharge) complications.
\end{abstract}

Materials \& methods: This prospective study was carried out in National Institute of Cardiovascular Diseases (NICVD), Dhaka during the period of June,2006 to May,2007. After fulfilling the exclusion criteria, 100 consecutive patients were selected as sample those underwent percutaneous coronary intervention. C-Reactive protein (CRP) was measured in all patients before the procedure. Those having pre-procedural CRP level <.3 mg/dl was labeled as group-I and those with CRP level e".3 $\mathrm{mg} / \mathrm{dl}$ was labeled as group-II. In hospital outcomes were compared between two groups after percutaneous coronary intervention.

Results: During the procedure, higher rate of complications (10.0\% vs 26.0\%) were noted in groupII, which was statistically significant. Among many complications abrupt occlusion, threatened (dissection and new thrombus) and residual stenosis were significantly high in group-II. Other complications like coronary spasm, arrhythmia, side branch occlusion, heart failure, shock and no reflow were also high in higher level of CRP level patients but statistically not significant. Inhospital complications were also significantly high (10.0\% vs $28.0 \%)$ in patients with higher level of CRP ie. Group-II. Among other complications recurrence of ischemia and myocardial infarction developed at significantly higher rate in group-II. Post procedural CK-MB rose significantly (18.0\% vs $36.0 \%$ ) in patients with higher level of CRP (group-II) in comparison to normal level of CRP patients (group-I). When the in-hospital complications were distributed among three tertiles of CRP, it was observed that complications were proportionately high with increasing level of CRP.

Conclusions: Many studies, conducted abroad, have suggested that factors like gender, extreme age, diabetes, multi-vessel disease and lesion characteristics adversely influence PCI outcome. But in this study, multivariate regression analysis has shown that elevated level of CRP is even stronger independent predictor of PCI outcome.

(Cardiovasc. j. 2011; 4(1): 32-41)

\section{Introduction:}

Coronary artery disease remains the leading cause of death worldwide in spite of relentless effort and research and by the year 2020 it will hold first place in the World Health Organization's list of leading cause of disability. ${ }^{1}$ It is one of the leading causes of death in the developed world as well as in the developing countries like Bangladesh. National data on incidence and mortality of coronary heart disease are few in Bangladesh. The incidence of coronary artery disease has increased from 3.3 per thousand to 14 per thousand from the year 1975 to $1985 .^{2}$

Major underlying mechanism of ischemic heart disease is atherosclerotic process in coronary arteries of heart. Atherosclerosis is a complex inflammatory-fibroproliferative response to deposition of plasma- derived atherogenic lipoprotein in the arterial intima (Falk \& Fuster 2001). ${ }^{3}$ Acute coronary syndrome has commonly been shown to result from disruption of 
atherosclerotic plaque at a site of a high density of inflammatory cells. ${ }^{4}$ Inflammation plays an important role in the initiation and progression of atherosclerosis by generating systemic blood markers of inflammation including C-reactive protein and proinflammatory cytokines, which in turn promote leukocyte, endothelial and smooth muscle cell activation, resulting in an increase in procoagulant activity, metalloproteinase release and neointimal proliferation. ${ }^{5}$ So it has emerged as powerful predictors of coronary events. ${ }^{6}$ Even small elevation of CRP within or just beyond the normal range, have been found to strongly predict future cardiovascular events in almost all studies. ${ }^{7}$

Percutaneous coronary intervention (PCI) has continued expanding its role in the management of coronary atherosclerosis. Though much progress has been made with PCI, since its first inauguration by Andreas Gruntzig in 1977, its glory in many cases is thwarted by some adverse complications. To accurately stratify patients according to their risk of future adverse events, a quest for risk predictors is ongoing worldwide, but only a few powerful and independent predictors of early and late major adverse cardiovascular events have been found. These include traditional risk factors, such as acute coronary instability, diabetes mellitus, reference vessel diameter, and lesion and/or stent length. ${ }^{8}$ Nonetheless, risk assessment is still incomplete if based only on such "traditional" prognostic variables. ${ }^{9}$

For this reason, since the last decade, biochemical markers have been carefully investigated; among these, several have been related to the risk of death, myocardial infarction, or restenosis after PCI, but only C-reactive protein (CRP) has been consistently shown to significantly and independently confer an adverse prognosis in patients with coronary artery disease, including those undergoing PCI. ${ }^{10}$ This is probably because of its relatively long half-life and chemical stability as well as the limited costs. ${ }^{11}$

There are many studies to show the late complication; ${ }^{12,13}$ but there are few studies as well, which have provided consistent and compelling association of pre-procedural CRP with early complications. ${ }^{14,15}$ We conducted this study to correlate the level of CRP with in-hospital adverse outcome in patients undergoing PCI and to assess the procedural and clinical success of PCI in patients with elevated CRP level.

Our hypothesis was - high baseline C-Reactive Protein level independently predicts in-hospital adverse outcome after elective PCI.

\section{Materials \& Methods:}

It was a $\mathrm{n}$ observational study conducted in Department of Cardiology,National Institute of Cardio-vascular Diseases, Dhaka, from January 2006 to December 2006. Patients who underwent percutaneous coronary intervention of native coronary arteries were considered for study. Patient with MI, multi vessel PTCA, total occlusion, previous PTCA or bypass surgery, left ventricular $\mathrm{EF}<30 \%$, recurrent inflammatory conditions known to increase CRP level or serum Creatinine $>2.5 \mathrm{mg} / \mathrm{dl}$ were excluded from the study.

Considering inclusion and exclusion criteria consecutive 50 patients underwent percutaneous coronary intervention with CRP level $<0.3 \mathrm{mg} / \mathrm{dl}$ and 50 angiographically matched patients with CRP level e"0.3mg/dl were studied.Patients with CRP level $<0.3 \mathrm{mg} / \mathrm{dl}$ constituted Group-I and patients with CRP level e" $0.3 \mathrm{mg} / \mathrm{dl}$ Group-II. The study protocol was approved by the institutional review board. Informed consent was taken from each patient or from the legal guardian. All patients were informed about the study. Data were collected in an approved data collected form.

\section{Pre-procedural work-up:}

Detailed history was taken, and clinical examination was performed.Baseline investigations like CBC, 12-lead ECG, chest X-ray, blood sugar, blood urea, serum creatinine, serum lipid profile, bleeding time, clotting time, VDRL test, HBsAg, HIV test, Anti-HBC and echocardiography were done. Elevated level of CRP was defined as the preprocedural CRP e" $0.3 \mathrm{mg} /$ dl. To show whether the rate of complication is directly related with the level of CRP, the level of CRP was again divided in to three tertile.

Tertile I- $\leq .3 \mathrm{mg} / \mathrm{dl}$, Tertile II- .3-1 mg/dl, Tertile III- $>1 \mathrm{mg} / \mathrm{dl}$.

Demographic profile, including age, sex, height, weight, body mass index (BMI) of both the groups, were noted. Risk factor profile included 
hypertension, diabetes, dyslipidaemia, smoking and family history of coronary artery disease (CAD). Echocardiographic variables specially included left ventricular ejection fraction (LVEF).

Pre-procedural angiographic variables including number of stenosed vessel (single

/double /triple), degree of stenosis (0 to 100\%) and type of lesion (ACC/AHA low-risk, intermediaterisk and high-risk lesions) were noted.

\section{Observation during the procedure:}

Procedural observations included observation of no reflow, slow reflow, side branch occlusion, residual stenosis, distal TIMI flow and immediate complications (e.g. abrupt occlusion, threatened occlusion, dissection, perforation, cardiogenic shock, significant arrhythmias, cardiac arrest, coronary artery spasm, emergency CABG and death).

\section{Post-procedural evaluation:}

All patients were observed meticulously during their hospital stay to see: Chest pain, Significant arrhythmia, Congestive cardiac failure, Cardiogenic shock, Emergency coronary artery bypass graft (CABG) surgery, CVA, Thromboembolism, Death.

Post- procedural follow-up began immediately after completion of the intervention itself, and was done hourly for the first 6 hours and then every morning and evening, or even more frequently if indicated. Subjective evidence of chest pain, breathlessness and pain in the limb through which vascular access was made were looked for. Pulse, blood pressure, chest, peripheral pulses and vascular access site were examined. Bed-side monitor parameters were noted and available medical records were scrutinized.

Twelve-lead ECGs were done: Immediately and twenty-four hours after the procedure, before discharge, and as necessary. CK-MB level in blood was measured six hours after the procedure and as necessary. All patients were followed- up throughout the period of index hospitalization till discharge or death.

\section{Estimation of C-Reactive Protein:}

We estimated High sensitive C-Reactive Protein (hs-CRP) by Immulite 2000 (DPC)/Vitros ECi
System (J\&J)/Abbott AxSym System Random Access Multibatch Immunoassay Analyzer.

\section{Statistical analysis:}

Data were collected in a pre-designed form. The data obtained, were expressed in frequency, percentage, mean \pm standard deviation, as applicable. Comparison between groups was done by chi-square test, Student's $t$ test, Fisher's exact test or others, as applicable.All data were analyzed by using computer-based SPSS (Statistical Program for Social Science) program, version 11.5.

\section{Observations and Results:}

A total of 100 consecutive patients were studied who underwent PTCA with stent. The main objectives of the study was to evaluate the predictive value of pre-procedural C-Reactive Protein on in-hospital (peri-procedural and predischarge) outcome after Percutaneous Coronary Intervention (PCI).

\section{Table-I}

Age and sex distribution of the study patients

\begin{tabular}{lcccc}
\hline Variables & \multicolumn{2}{c}{ Study subjects } & $\begin{array}{c}\text { Total } \\
(\mathrm{n}=100)\end{array}$ & P value \\
\cline { 2 - 3 } & $\begin{array}{c}\text { Group I } \\
(\mathrm{n}=50)\end{array}$ & $\begin{array}{c}\text { Group II } \\
(\mathrm{n}=50)\end{array}$ & & \\
\hline Age in years & & & & \\
$30-39$ & $7(14.0)$ & $5(10.0)$ & $12(12.0)$ & $0.538^{\mathrm{ns}}$ \\
$40-49$ & $19(38.0)$ & $20(40.0)$ & $37(37.0)$ & $0.837^{\mathrm{ns}}$ \\
$50-59$ & $19(38.0)$ & $18(36.0)$ & $39(39.0)$ & $0.835^{\mathrm{ns}}$ \\
$60-70$ & $5(10.0)$ & $7(14.0)$ & $12(12.0)$ & $0.538^{\mathrm{ns}}$ \\
Sex & & & & \\
Male & $45(90.0)$ & $46(92.0)$ & $91(91.0)$ & $0.728^{\mathrm{ns}}$ \\
Female & $5(10.0)$ & $4(8.0)$ & $9(09.0)$ & \\
\hline
\end{tabular}

N.B. Figure in parenthesis indicate percentages. Group I= hs-CRP level $<0.3 \mathrm{mg} / \mathrm{dl}$

Group II= hs-CRP level $\geq 0.3 \mathrm{mg} / \mathrm{dl}$. $\mathrm{P}$ value reached from chi-square test

Table I shows the age and sex distribution of the study subjects. In this study, the patients were grouped into two on the basis of hs-CRP level. The patients those who had hs-CRP level $<0.3 \mathrm{mg} / \mathrm{dl}$ considered as group I and those who had hs-CRP level e"0.3 mg/dl as group II. Then mean age of group I patients was $48.7 \pm 8.8$ years and $47.9 \pm 7.6$ years for group II patients. But the mean age difference was not statistically significant $(p>0.05)$. 
It was also found that among group I patients, $45(90.0 \%)$ were male and $5(10.0 \%)$ were female. Whereas among group II patients, 46(92.0\%) were male and $4(8.0 \%)$ were female. No statistically significant sex difference was found between two groups of patients ( $>00.05)$.

\section{Table-II}

Distribution of risk factors of the study patients

\begin{tabular}{cccc}
\hline Risk factors & \multicolumn{2}{c}{ Study subjects } & Total \\
\cline { 2 - 3 } & $\begin{array}{c}\text { Group I } \\
(\mathrm{n}=50)\end{array}$ & $\begin{array}{c}\text { Group II } \\
(\mathrm{n}=50)\end{array}$ & P value \\
\hline
\end{tabular}

Smoking

$\begin{array}{lrrrr}\text { Yes } & 34(68.0) & 39(78.0) & 73(73.0) & 0.260^{\text {ns }} \\ \text { No } & 16(32.0) & 11(22.0) & 27(27.0) & \end{array}$

Hypertension

$\begin{array}{lllll}\text { Yes } & 23(46.0) & 28(56.0) & 51(51.0) & 0.317^{\mathrm{ns}} \\ \text { No } & 27(54.0) & 22(44.0) & 49(49.0) & \end{array}$

Diabetes Mellitus

$\begin{array}{lllll}\text { Yes } & 19(38.0) & 26(52.0) & 45(45.0) & 0.159^{\text {ns }} \\ \text { No } & 31(62.0) & 24(48.0) & 55(55.0) & \end{array}$

Dyslipidaemia

\begin{tabular}{lllll} 
Yes & $11(22.0)$ & $13(26.0)$ & $24(24.0)$ & $0.639^{\mathrm{ns}}$ \\
No & $39(78.0)$ & $37(74.0)$ & $76(76.0)$ & \\
F/O IHD & & & & \\
Yes & $18(36.0)$ & $20(40.0)$ & $38(38.0)$ & $0.680^{\mathrm{ns}}$ \\
No & $32(64.0)$ & $30(60.0)$ & $62(62.0)$ & \\
\hline
\end{tabular}

N.B.-Figure in parenthesis indicate percentages, $\mathrm{P}$ value reached from chi-square test

Table II shows the percentage distribution of risk factors among the studied subjects. Among the group I patients, highest percentage were smokers 34 (68.0\%) followed by hypertension 23(46.0\%), diabetes mellitus 19(38.0\%), family history of IHD 18(36.0\%) and dyslipidaemia 11(22.0\%). Similarly in group II highest percentage were smokers $39(78.0 \%)$ followed by hypertension $28(56.0 \%)$, diabetes mellitus $26(52.0 \%)$, family history of IHD 20(40.0\%) and dyslipidaemia 13(26.0\%). No statistically significant difference was found between two groups of patients $(p>0.05)$ in terms of risk factors.
Table-III

Distribution of study subject according to category of coronary artery disease

\begin{tabular}{lcccc}
\hline CAD & \multicolumn{2}{c}{ Study subjects } & Total & P value \\
\cline { 2 - 3 } & $\begin{array}{c}\text { Group I } \\
(\mathrm{n}=50)\end{array}$ & $\begin{array}{c}\text { Group II } \\
(\mathrm{n}=50)\end{array}$ & & \\
\hline Stable angina & $26(52.0)$ & $20(40.0)$ & $46(46.0)$ & $0.130^{\mathrm{ns}}$ \\
Unstable & $24(48.0)$ & $30(60.0)$ & $54(54.0)$ & $0.629^{\mathrm{ns}}$ \\
angina & & & & \\
\hline
\end{tabular}

N.B.Figure in parenthesis indicate percentages, $P$ value reached from chi-square test

Table III shows that among group I patients highest percentage had stable angina $26(52.0 \%)$ followed by unstable angina $24(48.0 \%)$. Whereas in group II highest percentage had unstable angina $30(60 \%)$ followed by stable angina $20(40.0 \%)$. No statistically significant difference was found between two groups of patients ( $p>0.05)$.

\section{Table-IV \\ Distribution of study subjects according to ejection fraction.}

\begin{tabular}{|c|c|c|c|c|}
\hline \multirow{2}{*}{$\begin{array}{l}\text { Ejection } \\
\text { fraction }\end{array}$} & \multicolumn{2}{|c|}{ Study subjects } & \multirow{2}{*}{$\begin{array}{c}\text { Total } \\
(n=100)\end{array}$} & \multirow[t]{2}{*}{$\mathrm{P}$ value } \\
\hline & $\begin{array}{l}\text { Group I } \\
(\mathrm{n}=50)\end{array}$ & $\begin{array}{c}\text { Group II } \\
(\mathrm{n}=50)\end{array}$ & & \\
\hline $30-40$ & $2(4.0)$ & $6(12.0)$ & $8(8.0)$ & ${ }^{\mathrm{a}} 0.140^{\mathrm{ns}}$ \\
\hline $40-50$ & $12(24.0)$ & $12(24.0)$ & $24(24.0)$ & - \\
\hline $50-60$ & $20(40.0)$ & $21(42.0)$ & $41(41.0)$ & ${ }^{\mathrm{b}} 0.838^{\mathrm{ns}}$ \\
\hline$\geq 60$ & $16(32.0)$ & $11(22.0)$ & $37(37.0)$ & ${ }^{\mathrm{b}} 0.260^{\mathrm{ns}}$ \\
\hline
\end{tabular}

Table IV shows the distribution of study subjects by mean percent of ejection fraction. It was found that in group I, highest percentage was $20(40.0 \%)$ in the range of $50-60 \%$ followed by $16(32.0 \%)$ in e"60\%, 12(24.0\%) in 40-50\% and lowest 2(4.0\%) in the range of below $30-40 \%$. Similar pattern of ejection fraction was observed among group II patients with highest percentage $21(42.0 \%)$ was in the range of $50-60 \%$ followed by $12(24.0 \%)$ in the range of $40-50 \%, 11(22.0 \%)$ in e" $60 \%$ and $6(12.0 \%)$ in $30-40 \%$. No statistically significant difference was found between two groups of patients in terms of percent of ejection fraction ( $>>0.05)$ although mean percent of ejection fraction was higher in

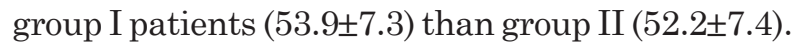


Table-V

Distribution of study subjects according to vessel involvement

\begin{tabular}{lcccc}
\hline Vessel & \multicolumn{2}{c}{ Study Subjects } & Total & p value \\
\cline { 2 - 3 } Involved & $\begin{array}{c}\text { Group I } \\
(\mathrm{n}=50)\end{array}$ & $\begin{array}{c}\text { Group II } \\
(\mathrm{n}=50)\end{array}$ & & \\
\hline Single & $24(48.0)$ & $18(36.0)$ & $42(42.0)$ & 0.224 \\
Double & $21(42.0)$ & $19(38.0)$ & $40(40.0)$ & 0.683 \\
Triple & $5(10.0)$ & $13(26.0)$ & $18(18.0)$ & 0.037 \\
\hline
\end{tabular}

N.B. Figure in parenthesis indicate percentages, $P$ value reached from chi-square test

Table V shows the percentage distribution of coronary arterial involvement disease by category of study subjects. It was found in group I patients, $24(48.0 \%)$ had single vessel disease, 21 (42.0\%) double vessel disease and rest $5(10.0 \%)$ had triple vessel disease. Whereas in group II patients, highest percentage $19(38.0 \%)$ had double vessel disease followed by 18 (36.0\%) single vessel and rest $13(26.0 \%)$ had triple vessel disease. Statistically significant difference was found in triple vessel disease between two groups of patients and others were not significant $(\mathrm{p}>0.05)$.

Table-VI

Distribution of study population by balloon and stent inflation pressure and time

\begin{tabular}{|c|c|c|c|c|}
\hline \multirow[t]{2}{*}{$\overline{\text { Parameters }}$} & \multicolumn{2}{|c|}{ Study subjects } & \multirow{2}{*}{$\begin{array}{c}\text { Total } \\
(\mathrm{n}=100)\end{array}$} & \multirow[t]{2}{*}{ p value } \\
\hline & $\begin{array}{l}\text { Group I } \\
(\mathrm{n}=50)\end{array}$ & $\begin{array}{c}\text { Group II } \\
(\mathrm{n}=50)\end{array}$ & & \\
\hline $\begin{array}{l}\text { PTCA balloon } \\
\text { inflation pressur } \\
\text { (atm) }\end{array}$ & $9.6 \pm 1.1$ & $9.9 \pm 1.0$ & $9.7 \pm 1.0$ & $0.177^{\mathrm{ns}}$ \\
\hline $\begin{array}{l}\text { PTCA balloon } \\
\text { inflation time (se }\end{array}$ & $\begin{array}{l}32.8 \pm 9.5 \\
\text { c) }\end{array}$ & $33.6 \pm 6.3$ & $32.9 \pm 7.9$ & $0.273^{\mathrm{ns}}$ \\
\hline $\begin{array}{l}\text { Stent inflation } \\
\text { pressure (atm) }\end{array}$ & $10.6 \pm 0.7$ & $10.9 \pm 1.1$ & $10.8 \pm 1.1$ & $0.399^{\mathrm{ns}}$ \\
\hline $\begin{array}{l}\text { Stent inflation } \\
\text { time }(\mathrm{sec})\end{array}$ & $32.9 \pm 8.6$ & $33.5 \pm 6.3$ & $33.2 \pm 7.5$ & $0.079^{\mathrm{ns}}$ \\
\hline
\end{tabular}

N.B.P value reached from unpaired student's t test

Table VI shows the inflation pressure and its duration for the intervention. It was found that no statistically significant mean difference was found between two groups of patients in terms of inflation pressure and its duration $(\mathrm{p}>0.05)$.

\section{Table-VII}

Distribution of study subjects by level of CK-MB after intervention

\begin{tabular}{lcccc}
\hline CKMB & \multicolumn{2}{c}{ Study subjects } & Total & p value \\
\cline { 2 - 5 } U/L & $\begin{array}{c}\text { Group I } \\
(\mathrm{n}=50)\end{array}$ & $\begin{array}{c}\text { Group II } \\
(\mathrm{n}=50)\end{array}$ & & \\
\hline$<25$ & $41(82.0)$ & $32(64.0)$ & $73(73.0)$ & $0.043^{\mathrm{s}}$ \\
25 & $9(18.0)$ & $18(36.0)$ & $27(27.0)$ & \\
\hline
\end{tabular}

N.B.Figure in parenthesis indicate percentages. $P$ value reached from chi square test

Table VII shows the distribution of study subjects by level of CKMB level at 6 hours after intervention. It was found that among group I subjects, 41(82.0\%) had CKMB level below $25 \mathrm{U} / \mathrm{L}$ and $9(18.0 \%)$ had above $25 \mathrm{U} / \mathrm{L}$ whereas among group II subjects, CKMB level was observed with highest percentage $32(64.0 \%)$ below $25 \mathrm{U} / \mathrm{L}$ and 18(36.0\%) above25 U/L and the difference was statistically significant $(\mathrm{p}<0.05)$ indicating group II patients had higher level of CKMB rise (36.0\%) compared with group I (18.0\%).

\section{Table-VIII}

\section{$\geq$ Distribution of study subjects by procedural complications}

\begin{tabular}{|c|c|c|c|c|}
\hline \multirow{2}{*}{$\begin{array}{l}\text { Procedural } \\
\text { complications }\end{array}$} & \multicolumn{2}{|c|}{ Study subjects } & \multirow{2}{*}{$\begin{array}{c}\text { Total } \\
(\mathrm{n}=100)\end{array}$} & \multirow[t]{2}{*}{$\bar{p}$ value } \\
\hline & $\begin{array}{l}\text { Group I } \\
(\mathrm{n}=50)\end{array}$ & $\begin{array}{c}\text { Group II } \\
(\mathrm{n}=50)\end{array}$ & & \\
\hline No complications & $45(90.0)$ & $37(74.0)$ & $82(82.0)$ & ${ }^{\mathrm{a}} 0.037^{\mathrm{s}}$ \\
\hline Abrupt occlusion & $1(2.0)$ & $7(14.0)$ & $8(8.0)$ & ${ }^{\mathrm{b}} 0.029^{\mathrm{s}}$ \\
\hline $\begin{array}{l}\text { Threatened } \\
\text { occlusion } \\
\text { (Dissection + } \\
\text { new thrombus) }\end{array}$ & $0(0.0)$ & & $6(6.0)$ & $\mathrm{b}_{0} 027 \mathrm{~s}$ \\
\hline Coronary spasm & $2(4.0)$ & $6(12.0)$ & $8(8.0)$ & ${ }^{\mathrm{b}} 0.268^{\mathrm{ns}}$ \\
\hline Arr & $1(2.0)$ & 4( & $5(5.0)$ & $\mathrm{b}^{\mathrm{b}} 0.362^{\mathrm{ns}}$ \\
\hline $\begin{array}{l}\text { Side branch } \\
\text { occlusion }\end{array}$ & $2(4.0)$ & $4(8.0)$ & $6(6.0)$ & $0.378^{\mathrm{ns}}$ \\
\hline Heart failure & $1(2.0)$ & $4(8$. & $5(5.0)$ & $0.362^{\mathrm{ns}}$ \\
\hline he & $0(0$. & 1( & $1(1.0)$ & $0.500^{\mathrm{ns}}$ \\
\hline ior & $0(0$. & $0(0.0)$ & $0(0.0)$ & \\
\hline No & $1(2.0)$ & $3(6$ & $4(4.0)$ & $0.617^{\mathrm{ns}}$ \\
\hline flow & $2(4$ & $7(14.0)$ & $9(9.0)$ & $0.159^{\text {ns }}$ \\
\hline $\begin{array}{l}\text { Residual } \\
\text { stenosis }\left(>30^{\circ}\right.\end{array}$ & $1(2.0)$ & $7(14.0)$ & $8(8.0)$ & $0.029^{\mathrm{s}}$ \\
\hline Emer & $0(0$ & (0.0) & $0(0.0)$ & \\
\hline Death & $0(0.0)$ & $0(0.0)$ & $0(0.0)$ & \\
\hline
\end{tabular}

N.B. Figure in parenthesis indicate percentages . ${ }^{\text {aP }}$ value reached from chi square test

${ }^{\mathrm{b}} \mathrm{P}$ value reached from Fisher's exact test 
Table VIII shows the procedural complications of the study subjects. It was found that $82(82.0 \%)$ of the study subjects had no procedural complications in the coronary blood vessels. Only $18(18.0 \%)$ developed complications, which is statistically significantly higher in group II $(\mathrm{p}<0.05)$. In group II number of abrupt occlusion was $7(14.0 \%)$ but in group I it was only one (2.0\%). Similar incidence was observed for slow reflow [group II 7(14.0\%) vs group I $2(4.0 \%)$ ] in two groups. Threatened occlusion $6(12.0 \%)$ and coronary spasm $6(12.0 \%)$ in group II were also distinctly higher than group I though statistically not significant. Other complications like arrhythmia [4(8.0\%) vs $1(2.0 \%)]$, side branch occlusion [4(8.0\%) vs $2(4.0 \%)]$, heart failure [[4(8.0\%) vs $2(4.0 \%)]$, no reflow [3(6.0\%) vs $1(2.0 \%)]$, hypotension/shock [1(2.0\%) vs $0(0.0 \%)]$ were oviously higher in group II but statistically not significant $(p>0.05)$. Complications like perforation, emergency $\mathrm{CABG}$ and death were nil in the both the groups.

\section{Table-IX}

Distribution of procedural complications with increasing tertile of CRP.

\begin{tabular}{|c|c|c|c|c|}
\hline $\begin{array}{l}\text { Procedural } \\
\text { complications }\end{array}$ & $\begin{array}{c}\text { Tertile I } \\
(\mathrm{n}=50) \\
<0.3 \\
\mathrm{mg} / \mathrm{dl} \\
\end{array}$ & $\begin{array}{c}\text { Tertile II }{ }^{r} \\
(\mathrm{n}=21) \\
0.3-1 \\
\mathrm{mg} / \mathrm{dl} \\
\end{array}$ & $\begin{array}{l}\text { Tertile III } \\
\begin{array}{c}(\mathrm{n}=29) \\
>1 \\
\mathrm{mg} / \mathrm{dl} \\
\end{array}\end{array}$ & $\begin{array}{l}\mathrm{P} \\
\text { value }\end{array}$ \\
\hline Abrupt occlusion & $1(2.0)$ & $2(9.5)$ & $5(17.2)$ & $0.029^{\mathrm{s}}$ \\
\hline $\begin{array}{l}\text { Threatened } \\
\text { occlusion (Dissectio } \\
\text { + new thrombus) }\end{array}$ & $\begin{array}{l}0(0.0) \\
\text { on }\end{array}$ & $1(4.8)$ & $5(17.2)$ & $0.027^{\mathrm{s}}$ \\
\hline Coronary spasm & $2(4.0)$ & $2(9.5)$ & $4(13.8)$ & $0.268^{\text {ns }}$ \\
\hline Arrhy & $1(2.0)$ & $1(4.8)$ & $3(10.3)$ & $0.362^{\text {ns }}$ \\
\hline $\begin{array}{l}\text { Side branch } \\
\text { occlusion }\end{array}$ & $2(4.0)$ & $2(9.5)$ & $3(10.3)$ & $0.378^{\mathrm{ns}}$ \\
\hline Heart failure & $1(2.0)$ & $1(4.8)$ & $3(10.3)$ & $0.362^{\text {ns }}$ \\
\hline Shock & $0(0.0)$ & $0(0.0)$ & $1(3.4)$ & $0.500^{\mathrm{ns}}$ \\
\hline Perforation & $0(0.0)$ & $2(9.5)$ & $5(17.2)$ & - \\
\hline No reflow & $1(2.0)$ & $0(0.0)$ & $3(10.3)$ & $0.617^{\mathrm{ns}}$ \\
\hline Slow flc & $2(4.0)$ & & $0(0.0)$ & $0.159^{\text {ns }}$ \\
\hline $\begin{array}{l}\text { Residual stenosis } \\
(>30 \%)\end{array}$ & $1(2.0)$ & $2(9.5)$ & $6(20.7)$ & $0.029^{\mathrm{s}}$ \\
\hline Emergency CABG & $0(0.0)$ & $0(0.0)$ & $0(0.0)$ & - \\
\hline Death & $0(0.0)$ & $0(0.0)$ & $0(0.0)$ & - \\
\hline
\end{tabular}

Analysis of the above table indicates statistically significant number of abrupt occlusion and threatened occlusion occurred in tertile II and tertile III in comparison to tertile I. Other complications are also high in tertile III. It is observed that complications are proportionately high according to increasing tertile of CRP.

\section{Table-X}

Distribution of study subjects according to Inhospital complications.

\begin{tabular}{|c|c|c|c|c|}
\hline \multirow[t]{2}{*}{ Complications } & \multicolumn{2}{|c|}{ Study subjects } & \multirow{2}{*}{$\begin{array}{c}\text { Total } \\
(\mathrm{n}=100)\end{array}$} & \multirow{2}{*}{$\begin{array}{c}\mathrm{p} \\
\text { value }\end{array}$} \\
\hline & $\begin{array}{c}\text { Group I } \\
(\mathrm{n}=50)\end{array}$ & $\begin{array}{c}\text { Group II } \\
(\mathrm{n}=50)\end{array}$ & & \\
\hline No complication & $45(90.0)$ & $36(72.0)$ & $87(87.0)$ & $0.022^{\mathrm{s}}$ \\
\hline \multicolumn{5}{|l|}{ ischaemia (ST, } \\
\hline \multicolumn{5}{|l|}{ T change) } \\
\hline Myocardial & $0(0.0)$ & $5(10.0)$ & $5(5.0)$ & $0.028^{\mathrm{s}}$ \\
\hline \multicolumn{5}{|l|}{ infarction (NSTEMI } \\
\hline \multicolumn{5}{|l|}{ + STEMI) } \\
\hline Hypotension/shock & $1(2.0)$ & $3(6.0)$ & $4(4.0)$ & $0.617^{\mathrm{ns}}$ \\
\hline Arrhythmia & $1(2.0)$ & $4(8.0)$ & $5(5.0)$ & $0.362^{\text {ns }}$ \\
\hline Heart failure & $1(2.0)$ & $4(8.0)$ & $5(5.0)$ & $0.362^{\mathrm{ns}}$ \\
\hline CVA & $0(0.0)$ & $0(0.0)$ & $0(0.0)$ & - \\
\hline Thrombo-embolism & $\mathrm{n} 0(0.0)$ & $0(0.0)$ & $0(0.0)$ & - \\
\hline CABG & $0(0.0)$ & $0(0.0)$ & $0(0.0)$ & - \\
\hline Death & $0(0.0)$ & $0(0.0)$ & $0(0.0)$ & - \\
\hline
\end{tabular}

Table X shows the in-hospital complications of the study subjects. It was found that the in-hospital complications was significantly high among the patients of group II $(\mathrm{p}<0.05)$. Among the patients of group I, (90.0\%) of the patients had no complications, whereas among group II, (72.0\%) had no complications. The most frequents complications among group I patients were recurrence of ischaemia (ST, T change) (4.0\%) followed by hypotension/shock, arrhythmia, heart failure $(2.0 \%$ each), whereas among group II patients, the most frequent complications were recurrence of ischaemia (ST, T change) (16.0\%) followed by arrhythmia, heart failure (8.0\%), myocardial infarction (NSTMI+STEMI) (6.0\%), hypotension/shock (4.0\%) etc. Statistically significant $(p<0.05)$ number of major adverse cardiac event occurred in group II 5(10.0\%) compared to group I $0(0.0 \%)$. 
Table-XI

Distribution of in-hospital complications with increasing tertile of CRP.

\begin{tabular}{lcccc}
\hline Complications & $\begin{array}{c}\text { Tertile I } \\
(\mathrm{n}=50) \\
<0.3\end{array}$ & $\begin{array}{c}\text { Tertile II Tertile III } \\
(\mathrm{n}=21)\end{array}$ & $\begin{array}{c}\mathrm{P} \\
(\mathrm{n}=29)\end{array}$ & value \\
& $\mathrm{mg} / \mathrm{dl}$ & $\mathrm{mg} / \mathrm{dl}$ & $\mathrm{mg} / \mathrm{dl}$ & \\
\hline $\begin{array}{l}\text { Recurrence of } \\
\text { ischaemia (ST, }\end{array}$ & & & & \\
$\begin{array}{l}\text { T change) } \\
\text { Myocardial }\end{array}$ & $2(4.0)$ & $1(4.8)$ & $6(20.7)$ & $0.045^{\mathrm{s}}$ \\
infarction (NSTEMI & & & & \\
+ STEMI) & $0(0.0)$ & $1(4.8)$ & $4(13.8)$ & $0.028^{\mathrm{s}}$ \\
Hypotension/shock & $1(2.0)$ & $1(4.8)$ & $2(6.9)$ & $0.557^{\mathrm{ns}}$ \\
Arrhythmia & $1(2.0)$ & $1(4.8)$ & $3(10.3)$ & $0.362^{\mathrm{ns}}$ \\
Heart failure & $1(2.0)$ & $1(4.8)$ & $3(10.3)$ & $0.362^{\mathrm{ns}}$ \\
CVA & $0(0.0)$ & $0(0.0)$ & $0(0.0)$ & - \\
Thrombo-embolism & $0(0.0)$ & $0(0.0)$ & $0(0.0)$ & - \\
CABG & $0(0.0)$ & $0(0.0)$ & $0(0.0)$ & - \\
Death & $0(0.0)$ & $0(0.0)$ & $0(0.0)$ & - \\
\hline
\end{tabular}

Analysis of the above table indicates statistically significant number of recurrence of ischaemia and myocardial infarction (NSTEMI + STEMI) occurred in tertile II and tertile III in comparison to tertile I. Other complications like hypotension/shock, arrhythmia, heart failure, CVA, thromboembolism, CABG and death are also obviously higher in tertile III. It is observed that complications are almost proportionately high according to increasing tertile of CRP.

Table-XII

Logistic regression analysis of rise of $C R P$ (tertile I vs tertile II+III) with selected variables

\begin{tabular}{lll}
\hline Variables & $\begin{array}{l}\text { Odd Ratio } \\
\text { (95\% Confidence }\end{array}$ & P value \\
& Interval) & \\
\hline CRP tertiles (I vs II+III) & $3.45(1.31-8.58)$ & 0.013 \\
Diabetes mellitus & $3.28(1.32-7.833)$ & 0.045 \\
Age & $4.83(1.50-15.803)$ & 0.783 \\
Smoking & $1.43(0.76-2.66)$ & 0.277 \\
Unstable angina +MI & $5.80(1.20-27.80)$ & 0.025 \\
Systemic hypertension & $3.31(1.273-9.453)$ & 0.052 \\
\hline
\end{tabular}

To assess the role of independent role of CRP for prediction of complications, multivariate analysis was done. Table XII shows multivariate logistic regression analysis with high CRP (tertile II+III) as dependant variable and age, sex, HTN, smoking, diabetes, UA and MI as independent variable. It shows that elevated CRP having odd ratio of 3.45 - has got independent relationship with adverse outcome. It is also evident here that DM and UA and MI also have independent influence on PCI outcome.

\section{Discussion:}

In this prospective study, 100 patients were included out of 111 patients, who underwent percutaneous coronary intervention (PCI) in NICVD. Eleven of them were excluded because 3 of them underwent double vessel PCI, 2 had inflammatory disease, 3 had total occlusion and 2 had previous PTCA and another had serum creatinine $>2.5 \mathrm{mg} / \mathrm{dl}$. The study population was divided into two equal groups of 50 on the basis of pre-procedural CRP level. Patients having CRP level $<0.3 \mathrm{mg} / \mathrm{dl}$ were labeled as Group-I and e" $0.3 \mathrm{mg} / \mathrm{dl}$ were labeled as Group-II. Again on few important issues these patients were divided in three tertile depending on CRP level. To show the rate of complication is directly related with the level of CRP, the level of CRP was again divided in to three tertile. Tertile I- $<0.3 \mathrm{mg} / \mathrm{dl}$, tertile II-0.3$1 \mathrm{mg} / \mathrm{dl}$ and tertile III- $>1 \mathrm{mg} / \mathrm{dl}$.

In this study, the outcomes of PCI were observed in both the groups during and after the procedure as long as the patients remained in the hospital. The outcomes were determined in terms of angiographic, procedural and clinical success, as well as of associated complications during the aforementioned period.

The mean age of the study subjects was $48.2 \pm 8.1$ years with a range from 31 to 70 years. The mean age of group-I patients was $48.7 \pm 8.8$ years and $47.9 \pm 7.6$ years for group-II patients. But the mean age difference was not statistically significant $(p>0.05)$ between two groups. The age structure was consistent with Ahmed et $\mathrm{al}^{16}$ in a similar study showing angiographic severity in relation to CRP level. The majority (91\%) of the study population was male and remaining $9 \%$ were female. But no statistical significant sex difference was found between two groups of patients $(p>0.05)$. Similar pattern was noted by Rahman et al. ${ }^{17}$ The body mass index for group-I was $25 \pm 2.3$ and for 
group-II was 26.5 \pm 3.1 . The difference was not significant.

Regarding the risk factors, smoking was most prevalent (68.0\%) and (78.0\%) in group-I and groupII respectively, followed by hypertension $(46.0 \%$ vs $56.0 \%$ ), diabetes mellitus ( $19.0 \%$ vs $26.0 \%$ ), dyslilpidemia (22.0\% vs $26.0 \%)$ and family history of IHD (36.0\% vs 40.0\%) in group-I and Group-II respectively, but no statistically significant difference was observed between two groups. Similar pattern of risk factors was found by Rahman et al. ${ }^{17}$ \& Ahmed et al. ${ }^{16}$ However the studies carried out abroad by Chew et al. ${ }^{15}$ and Buffon et al. ${ }^{14}$ demonstrated different pattern. This may be due to different ethnicity and socio-cultural background of their study population.

Different categories of coronary artery diseases were stable angina $(52.0 \%$ vs $40.0 \%)$, unstable angina ( $48.0 \%$ vs $60.0 \%$ ) respectively in group-I and group-II. Although stable angina was distinctly higher in group-I and unstable angina in group-II, no statistical difference was noted between two groups.

Mean percent of ejection fraction (EF\%) was $53.9 \pm 7.3 \mathrm{n}$ group-I and mean percent of ejection fraction $52.2 \pm 7.4$ in group-II. Although it was higher in group-I, no significance difference was marked. This observation is consistent with Chew et al. ${ }^{15}$

In terms of number of vessels involved, no significant difference was observed between groupI and group-II regarding single vessel $(48.0 \%$ vs $36.0 \%)$ and double vessel (42.0\% vs $38.0 \%)$ involvement, but statistically significant difference was found regarding triple vessel disease $(10.0 \%$ vs $26.0 \%$ ) between the two groups. It indicates patients with high CRP level have higher number of triple vessel disease.

No statistically significant ( $p>0.05$ ) difference was observed between group-I and group-II in term of type of lesion distribution, although type $\mathrm{C}$ lesion was obviously higher in group-II (16.0\% vs $28.0 \%)$. In this study it was found that type B lesion was the commonest type of lesion in both the groups. This finding is consistent with Rahman et al. ${ }^{17}$ In a study by Ahmed et al. ${ }^{16}$ it was observed that significantly higher percentage of complex lesion was in patients with higher CRP level.
In this study, PTCA balloon inflation pressure and time, stent inflation pressure and time did not show any statistically significant difference $(p>0.05)$ between two groups. Average PTCA balloon inflation pressure was $9.7 \pm 1.0 \mathrm{~atm}$. and stent inflation pressure was $10.8 \pm 1.1 \mathrm{~atm}$. PTCA balloon inflation time was $32.9 \pm 7.9$ and stent inflation time was $33.2 \pm 7.5$ seconds.

During the interventional procedure, as a whole, statistically significant $(p<0.05)$ number of complications occurred in group-II in comparison to group=I (46.0\% vs $10.0 \%)$. Among the statistically significant complications between two groups were abrupt occlusion (2.0\% vs $14.0 \%)$, threatened occlusion $(0.0 \% \mathrm{v} 14.0 \%)$ and residual stenosis (2.0\% vs $14.0 \%)$. Chew at el. also observed abrupt occlusion and threatened occlusion (dissection + new thrombus) in similar pattern (10.0\% vs $14.0 \%)$. Other complications like coronary spasm (4.0\% vs $16.0 \%)$ arrhythmia ( $2.0 \%$ vs $8.0 \%$ ), side branch occlusion ( $4.0 \%$ vs $8.0 \%$ ), heat failure $(2.0 \%$ vs $8.0 \%)$, shock $(0.0 \%$ vs $2.0 \%)$, no reflow (2.0\% vs $6.0 \%)$ and slow reflow (2.0\% vs .0\%) were obviously higher in group-II although statistically not significant. Emergency Coronary Artery Bypass Graft (CABG) and death were nil in both groups.

In hospital complications were also significantly high in group-II $(\mathrm{p}<0.05)$ in comparison to group-I (10.0\% vs $280 \%)$. Among the statistically significant complications were recurrence of ischemia $(4.0 \%$ vs $16.0 \%)$ and myocardial infarction $(0.0 \%$ vs $10.0 \%)$. The rate of complication is a bit higher than the findings by Chew et al. ${ }^{15}$ but there is no other study to compare the in-hospital complication rate with this study.

Other complications like hypotension $(2.0 \%$ vs $6.0 \%)$, arrhythmia (2.0\% vs $8.0 \%)$ and heart failure $(2.0 \%$ vs $8.0 \%)$ were distinctly higher in group-II but this difference are not statistically significant. CVA and other thrombo-embolism, cardiovascular accidents, CABG and death were nil in both groupI and group-II. In the study by Chew et al. ${ }^{15}$ death and myocardial infarction were observed in higher rate $(10.0 \%$ vs $16.8 \%)$ than this study. For this higher rate no correlation could be made other than increase prevalent of hypertension $(79.0 \% \mathrm{vs}$ $51.0 \%)$. But Chew et al. did not label hypertension as an independent risk factor for complication. 
Rahman et al. ${ }^{17}$ showed in his study significantly higher morbidity and mortality in patients with Acute Coronary Syndrome with higher CRP level.

When the in-hospital complications were distributed according to increasing tertile of CRP, it showed a proportionate increase of complications ie, highest rate $\mathrm{f}$ complications were observed in highest tertile (Tertile III) of CRP. This pattern of complications is quite consistent with that of the study by Chew et al, ${ }^{15}$ Winter et $\mathrm{al}^{18}$ and Buffon et al. ${ }^{14}$ Buffon et al $^{14}$ in his study showed that both procedural (abrupt occlusion and threatened abrupt occlusions) and in-hospital (MI and recurrence of ischemia) complications occurred in highest quartile of CRP level. Similar trend was observed in the study by Winter et al.

According to clinical diagnosis, patients with stable angina developed the highest procedural and inhospital complications (abrupt occlusion, threatened occlusion and in-hospital myocardial infarction) when they had CRP level in highest tertile ie. Tertile-III (2.0\% vs $8.0 \%)$. In case of MI and Unstable Angina rate of development of aforementioned complications are directly proportional to the CRP level. They also developed highest complication when their CRP level was in the highest tertile (1.0\% vs $12.0 \%)$. This observation is almost similar to that of Buffon et al. ${ }^{14}$

The patients with high CRP level ie. Group-II had longer post-interventional hospital stay than those with low level group ie. Group-I (3.3 \pm 2.6 vs $5.5 \pm 3.9$ ) and the difference between two groups are statistically significant $(p<0.05)$. No above mentioned studies showed this comparison. Anyway, higher incidence of in-hospital complications in higher CRP level group can explain this longer hospital stay.

Inconsistent and weak correlation with early complications following PTCA were reported for female gender \& diabetes by Ellis SG et al., ${ }^{19}$ extreme age by Thompson et al.(1993) ${ }^{20}$ multivessel disease by Gerald T. O'Connor et al. ${ }^{21}$ lesion characteristic by Tana et al. ${ }^{22}$ and White CJ et al. ${ }^{23}$ and hemostatic variables by F.C. Schoebela ${ }^{24}$ Evidence of intracoronary thrombus was found to be more consistent predictor of early adverse events, ${ }^{21,23}$ but its practical value is limited by its low prevalence in the baseline angiogram. ${ }^{23} \mathrm{In}$ clinical practice, unstable angina is the most important predictor of acute complication following PTCA $;{ }^{25}$ however, our observation that CRP levels are even stronger predictor of early adverse events suggests that the degree of activation of inflammatory cells is a more important determinant of early outcome after PTCA than clinical instability.

\section{Limitations of the study:}

Although the result of this study supports the hypothesis, yet it has got some limitations.

-This was a prospective, observational study, not randomized.

-Sample size was small.

-Only in-hospital observations was done, long term follow up could increase the strength of this study.

-It was conducted in a single center.

\section{Conclusion:}

The study reveals that $\mathrm{C}$-Reactive Protein level was a significant independent predictor of short term (procedural and pre-discharge) outcome following Percutaneous Coronary Intervention (PCI). Further clinical trials should be carried out to determine whether any measure like CRP reduction by statins, anti-inflammatory drugs or use of Gp IIb/IIIa receptor blocker could be helpful for patients with high pre-procedural level of CRP to reduce or prevent in hospital adverse outcome after PCI. Because of its relatively long half life and chemical stability as well as the limited costs and logistic hurdles faced by clinicians to measure it, CRP can be a very attractive and useful marker to accurately risk stratify patients before PCI.

\section{References:}

1. Murray CJ, Lopez AD. Global mortality, disability, and the contribution of risk factors: Global Burden of Disease Study. Lancet1997; 349(9063):1436-42

2. Malik, A, Islam, MN, Zafar, A, Khan, AK \& Ramizuddin, M. Clinical patters of ischemic heart disease and its association with some known risk factors. Bangladesh Heart Journal 1987; 2: 1-9.

3. Falk E, Shah PK, Fuster V. Coronary plaque disruption (Review). Circulation 1995; 92: 657-671.

4. AC van der Wal, AE Becker, CM van der Loos and PK Das. Site of intimal rupture or erosion of thrombosed coronary atherosclerotic plaques is characterized by an inflammatory process irrespective of the dominant plaque morphology. Circulation 1994;89:36-44. 
5. Cermak, J, Key, NS, Bach, RR, Balla, J, Jacob, HS \& Vercellotti, GM. C-reactive protein induces human peripheral blood monocytes to synthesize tissue factor. Blood 1993; 82 : 513-20.

6. Ridker, PM, Cushman, M, Stamper, MJ, Tracy, RP, Hennekens, CH. Inflammation, aspirin, and the risk of cardiovascular disease in apparently healthy men. $N$ Engl J Med 1997; 336: 973-9.

7. Danes, J, Collins, S, Appleby, P \& Peto, R.Association of fibrinogen, C- reactive protein, Albumin, and Leukocyte count with coronary heart disease; Meta-analysis of prospective studies. JAMA 1998; 279:1477-82.

8. Giuseppe G. L. Biondi-Zoccai, Antonio Abbate, Giovanna Liuzzo and Luigi M. Biasucci. Atherothrombosis, inflammation, and diabetes. J Am Coll Cardiol 2003; 41:1071-1077.

9. Kastrati, A, Schomig, A, Elezi, S, Schuhlen, H, Dirschinger, J, Hadamitzky, M.Predictive factors after coronary stent placement. J Am Coll Cardiol 1997; 30:1428-36.

10. Liuzzo, G, Biasucci, LM, Gallimore, JR, Grillo, RL, Rebuzzi, AG, Pepys, MB, Maseri, A. Prognostic value of C-reactive protein and plasma amyloid A protein in severe unstable angina. $N$ Engl $J$ Med 1994;331: 41724 .

11. Abbate, A, Biondi-Zoccai, GGL, Brugaletta, S, Liuzzo, G \& Biasucci ,LM. C-Reactive protein and other inflammatory biomarkers as predictors of outcome following acute coronary syndromes. Semin Vasc Med 2003; 3: 377-385.

12. De Winter, RJ, Koch, KT, van Straalen, JP, Heyde, G, Bax, M \& Schotborgh, CE. C-reactive protein and coronary events following percutaneous coronary angioplasty. Am J Med 2003; 115: 65-90.

13. Walter, DH, Fichtscherer, S, Sellwig, M, Auch-Schwelk, W, Schachinger, V \& Zeiher, AM.Preprocedural Creactive protein levels and cardiovascular events after coronary stent implantation. J Am Coll Cardiol 2001; 37: 839-46.

14. Buffon, A, Liuzzo, G, Biasucci, LM, Pasqualetti, P, Ramazzotti, V, Rebuzzi, AG, Crea, F, \& Maseri, A. Preprocedural serum levels of C-reactive protein predict early complication and late restenosis after angioplasty', J Am Coll Cardiol 1999; 34: 1512-21.

15. Chew, DP, Bhatt, DL, Robbin, MA, Penn, MS, Schneider, JP\& Lauer, MS. Incremental prognostic value of elevated baseline C-reactive protein among established markers of risk in percutaneous coronary intervention. Circulation 2001;103: 992-7.

16. Ahmed, M, Majumder, AAS, Haque SA \& Rahman S. Relationship of baseline white blood cell count and Creactive protein with angiographic severity of coronary artery disease in patients with acute coronary syndrome', Thesis, University of Dhaka, Bangladesh.2004.

17. Rahman, A, Islam, N, Malek F \& Faruque, M. Value of C-reactive protein on prognosis in patients with unstable angina Thesis, University of Dhaka, Bangladesh.2000.

18. Robbert J de Winter, Karel T Koch, Jan P van Straalen, Gerlind Heyde, et al. C-reactive protein and coronary events following percutaneous coronary angioplasty. The American Journal of Medicine 2003;115(2): 85-90.

19. SG Ellis, MJ Cowley, G DiSciascio, U Deligonul, EJ Topol, TM Bulle, and MG Vandormael. Determinants of 2-year outcome after coronary angioplasty in patients with multivessel disease on the basis of comprehensive preprocedural evaluation. Implications for patient selection. The Multivessel Angioplasty Prognosis Study Group. Circulation 1991;83:1905-1914.

20. RC Thompson, DR Holmes Jr, BJ Gersh and KR Bailey. Predicting early and intermediate-term outcome of coronary angioplasty in the elderly. Circulation 1993;88:1579-1587.

21. Gerald T. O'Connor, David J. Malenka, Hebe Quinton, John F. Robb, Mirle A. Kellett, Jr.,Samuel Shubrooks, William A. Bradley, Michael J. Hearne. Multivariate prediction of in-hospital mortality after percutaneous coronary interventions in 1994-1996. J Am Coll Cardiol 1999; 34:681-691

22. Kim Tana, , Neil Sulkea, Nick Taub and Edgar Sowton. Clinical and lesion Ellis SG et almorphologic determinants of coronary angioplasty success and complications: Current experience. J Am Coll Cardiol 1995, 25(4) 855-865

23. Christopher J. White. Angiographic predictors of adverse outcomes in the modern interventional era. $J$ Am Coll Cardiol 2003; 42:989-990.

24. F.C. Schoebela, A.J. Petersa, I. Kreisa, F. Gradausa, M. Heinsb and T.W. Jax. Relevance of hemostasis on restenosis in clinically stable patients undergoing elective PTCA. Thrombosis Research 2008; 122(2):229-236.

25. Eric D. Grassman, Ferdinand Leya, Sarah A. Johnson, Bruce E. Lewis, Mark W. Wolfe, John T. Strony, Burt Adelman and John A. Bittl. Percutaneous transluminal coronary angioplasty for unstable angina: Predictors of outcome in a multicenter study. Journal of Thrombosis and Thrombolysis 1994; 1(1): 73-78. 